BULL. AUSTRAL. MATH. SOC.

MOS 73605

VOL. $5(1971), 427-428$.

$(49020)$

\title{
Perturbation methods applied to the nonlinear symmetric bending of circular elastic plates
}

\author{
B.P. Garfoot
}

In this work, a refinement of the theory for the moderately large symmetrical deflections of circular and annular isotropic elastic plates is presented.

In order to obtain this refinement, the method of matched asymptotic expansions was applied to the full equilibrium equations of nonlinear elasticity with nonlinear stress-strain laws and the full nonlinear strain-displacement relations for the symmetrical bending of circular and annular plates. Six boundary conditions at the edges were considered.

The first two terms of the composite expansions for the solutions have been found for the following three plate theories:

(a) the large deflection theory,

(b) the 'linear stretching' theory, ana

(c) the 'linear bending' theory.

The first term of the interior expansions in each of these three theories coincides with the von Kármán theory of large deflections for (a), and the classical small-defiexion theory for (b) and (c).

In the large deflection theory, two boundary layer effects have been found; one due to the magnitude of the vertical load and the other due to the thinness of the plate. In the two 'linear' theories, only a thickness boundary layer effect occurs.

Received 22 June 1971. Thesis submitted to the University of Queensland, October 1970. Degree approved, June 1971. Supervisor: Dr V.G. Hart. 
A two parameter asymptotic expansion has been obtained for the large deflection of an annular plate under a particular set of boundary conditions. The method of multiple scales was used to show the effects of the load boundary layer.

An appendix contains the solution of a problem on the deformation of the semi-infinite strip. This problem is closely related to the analysis of the thickness boundary layer. 